\title{
A hypercoagulable state associated with intravenous pulse methylprednisolone therapy in patients with Graves' Orbitopathy
}

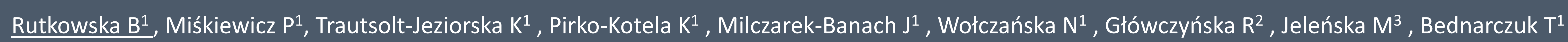
${ }^{1}$ Medical University of Warsaw, Department of Internal Medicine and Endocrinology ${ }^{2}$ Medical University of Warsaw, 1st Department of Cardiology ${ }^{3}$ Medical University of Warsaw, Department of General, Vascular and Transplant Surgery

\section{INTRODUCTION}

Chronic exposure to glucocorticoid (GCS) excess, either endogenous or exogenous, is associated with a hypercoagulable state and a high risk of thromboembolic complications like deep vein thrombosis and pulmonary embolism. The underlying mechanism of this phenomenon is still unclear. Chronic exposure of excess of GCS can cause disorders of all components of the Virchow triad, namely: i) abnormalities of hemostatic parameters, ii) vascular and endothelial damage secondary to obesity, insulin resistance, hypertension or dyslipidemia and iii) disturbed blood flow secondary to polycythemia, obesity or decreased mobility. However, there is a lack of studies dealing with the problem of influence of high dose intravenous (iv) GCS pulse therapy on coagulation system. This treatment is commonly used for a wide range of inflammatory, neoplastic and autoimmune diseases, including Graves' Orbitopathy (GO). In literature, there are some reported cases of even fatal side effects related to this therapy, including pulmonary embolism. Moreover, some of those diseases necessitating iv GCS treatment can cause a hypercoagulable state by itself. Taking the foregoing into account, it is important to determine whether the use of GCS enhances this process and whether thromboprophylaxis for patients receiving this kind of treatment should be recommended.

\section{AlM}

The aim of the study was to examine the influence of iv methylprednisolone (MP) on hemostasis in patients with active, moderate to severe GO. Patients were treated according to European Group on Graves' Orbitopathy (EUGOGO) recommendation with MP pulse given once a week for 12 consecutive weeks (cumulative dose $4.5 \mathrm{~g})$.

\section{DESCRIPTION OF METHODS}

\begin{tabular}{|c|c|}
\hline & Mean (ISD) or $n(\%)$ \\
\hline Patients & 20 \\
\hline Age & $54( \pm 11,4)$ \\
\hline Femele & $14(70 \%)$ \\
\hline Smoking & $11(55 \%)$ \\
\hline Hypertension & $7(35 \%)$ \\
\hline Dibetes mellitus & none \\
\hline Low-density lipoprotein (LDL) (<130 mg/dL) & $111,5( \pm 26,6)$ \\
\hline Body Mass Index (BMI) (kg/m²) & $24,5( \pm 4,1)$ \\
\hline Thyroid-stimulating hormone (TSH) (0.4 - 4.0 mIU/L) & $1,5( \pm 1,2)$ \\
\hline History of venous thromboembolism events & none \\
\hline Use of medications that affect hemostasis & none \\
\hline
\end{tabular}

Table 1. Characteristic of patients

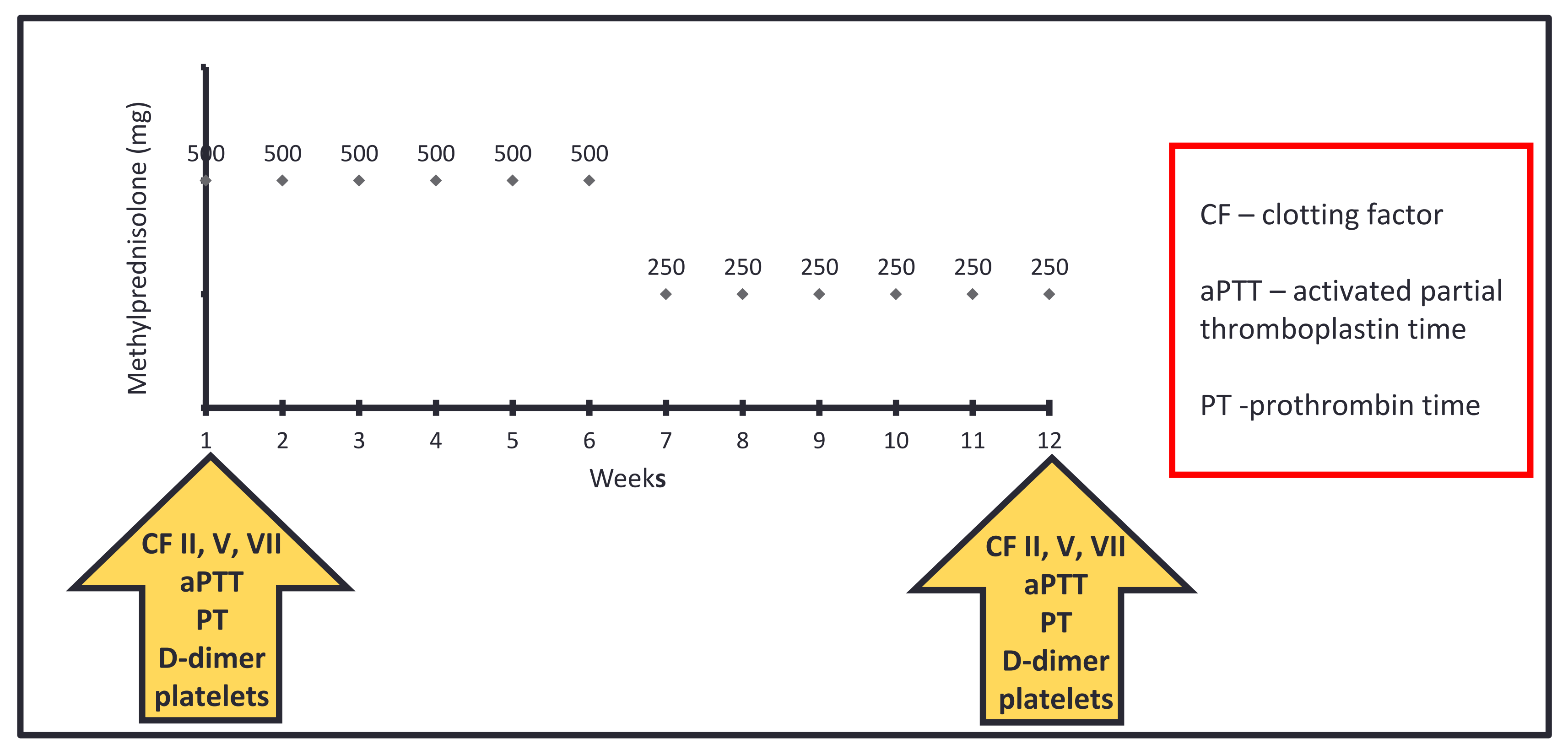

Figure 1. Schedule of treatment and interventions

\section{RESULTS}

Statistically significant increase of clotting factor (CF )II, CF V, CF VII and platelets was observed. The increase was noted respectively in $90 \%, 75 \%, 70 \%$ and $63 \%$ of patients.

$>$ Prothrombin time (PT) and activated partial thromboplastin time (aPTT) tended to decrease - in $65 \%$ and $50 \%$ of patients respectively, this change did not demonstrate statistical significance.

$>$ The increase of D-dimer in $55 \%$ of patients was observed, in $50 \%$ the increase was over $20 \%$.

$>$ There were no episodes of clinically overt venous thromboembolism.
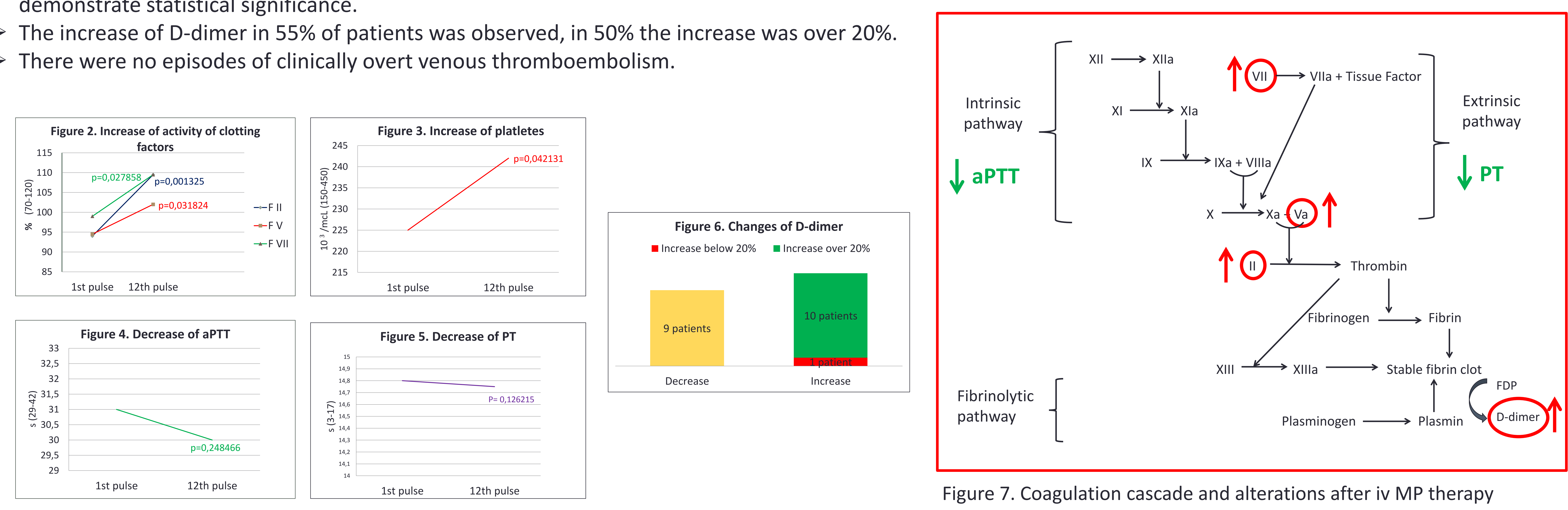

Figure 7. Coagulation cascade and alterations after iv MP therapy

\section{CONCLUSIONS}

High dose iv MP pulse therapy in patients with GO may be associated with a hypercoagulable state which can be a cause of fatal adverse effects (e.g. pulmonary embolism). This study indicates the increase of activity of CF II, CF V and CF VII with subsequent shortening of aPTT and PT. However, the casual relationship of those changes with the onset of clinically overt thrombosis remains to be confirmed. In patients with additional risk, prophylactic treatment may be required. 